\title{
Cerebral Coenurosis of a Long-Tailed Goral, Naemorhedus caudatus, in Korea
}

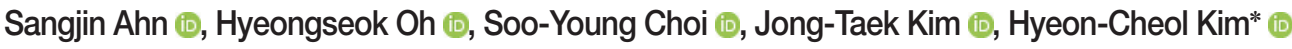 \\ College of Veterinary Medicine and Institute of Veterinary Science, Kangwon National University, Chuncheon 24341, Korea
}

\begin{abstract}
We intended to describe a case of cerebral coenurosis in a long-tailed goral, Naemorhedus caudatus, from Hwacheon-gun, Gangwon-do (Province), in the Korea. The goral, a 10-year-old male, was suffering from neurological symptoms, such as turning the circle to one side without lifting the head straight, and died at 30 days after admission to the wildlife medical rescue center in Chuncheon-si, Gangwon-do. A fluid-filled cyst was detected in the left cerebral hemisphere by computed tomography and magnetic resonance imaging. The cyst removed from the deceased goral was transparent, about $3 \times 3 \mathrm{~cm}$ in size, contained a clear fluid and approximately 320 protoscolices invaginating from the internal germinal layer. The protoscolex had 4 suckers and a rostellum with 28 hooklets arranged in 2 rows. By the present study, a case of cerebral coenurosis was first confirmed in a long-tailed goral, N. caudatus, from Gangwon-do, in Korea. The residents frequently exposed in the sylvatic environment should be careful the accidental infections of zoonotic metacestode of Taenia multiceps, Coenurus cerebralis, in Korea.
\end{abstract}

Key words: Coenurus cerebralis, Taenia multiceps, coenurosis, long-tailed goral

\section{INTRODUCTION}

Coenurosis is a zoonotic parasitic disease caused by the infections of metacestode of some Taenia species tapeworm, i.e., T. multiceps, T. brauni, T. serialis and T. glomeratus [1]. In particular, Coenurus cerebralis, the larval stage of T. multiceps mainly invades the central nervous system of sheep and goats, causing significant economic losses in their production [2]. Coenurus cerebralis penetrates the brain of the intermediate host, forming cysts, and the location and size of the cysts determine pathogenicity of coenurosis [3]. In addition, coenurosis is a zoonotic disease that can cause serious neurological problems in humans due to accidental infection [4]. In contrast, T. serialis, T. brauni and T. glomeratus infect with the soft/subcutaneous connective tissue, musculoskeletal system and visceral organs [5].

Identification of $C$. cerebralis is based on a combination of clinical symptoms, epidemiological events, hosts, anatomical location and morphological features [6]. Coenurosis is wide-

\footnotetext{
- Received 17 September 2020, revised 15 December 2020, accepted 28 December 2020. *Corresponding author (advs@kangwon.ac.kr) (C) 2021, Korean Society for Parasitology and Tropical Medicine This is an Open Access article distributed under the terms of the Creative Commons Attribution Non-Commercial License (https://creativecommons.org/licenses/by-nc/4.0) which permits unrestricted non-commercial use, distribution, and reproduction in any medium, provided the original work is properly cited.
}

spread throughout the world, including in Europe and the United States, Central Asia, especially in Africa and Southeast Asia, causing economic losses to the animal husbandry industry in many parts of the world $[7,8]$. However, in Korea, there have been no reports on the metacestode, Coenurus cerebralis, and adult of T. multiceps. Therefore, we intended to describe a case of cerebral coenurosis, which was detected in a long-tailed goral, N. caudatus, from Hwacheon-gun, Gangwon-do, Korea.

\section{CASE RECORD}

On October 5, 2019, a long-tailed goral was rescued at Hwacheon-gun, Gangwon-do (latitude 38.122320; longitude: $127.510020)$ and was brought to the Gangwon Wildlife Medical Rescue Center at Kangwon National University. At the time of rescue, the goral tilted his head to one side and did not move. It also showed typical clinical neurological signs such as failure to hold the head straight, torticollis, dullness, lethargy and disorientation. Based on the neurological symptoms of the goral, routine preliminary clinical tests, and radiographic examinations such as radiographs, computed tomography (CT), and magnetic resonance imaging (MRI) were performed. Goral was judged to be 10 years old based on the horn rings [9]. Gingival ulceration was detected by physical examination, and no other abnormalities were detected. 

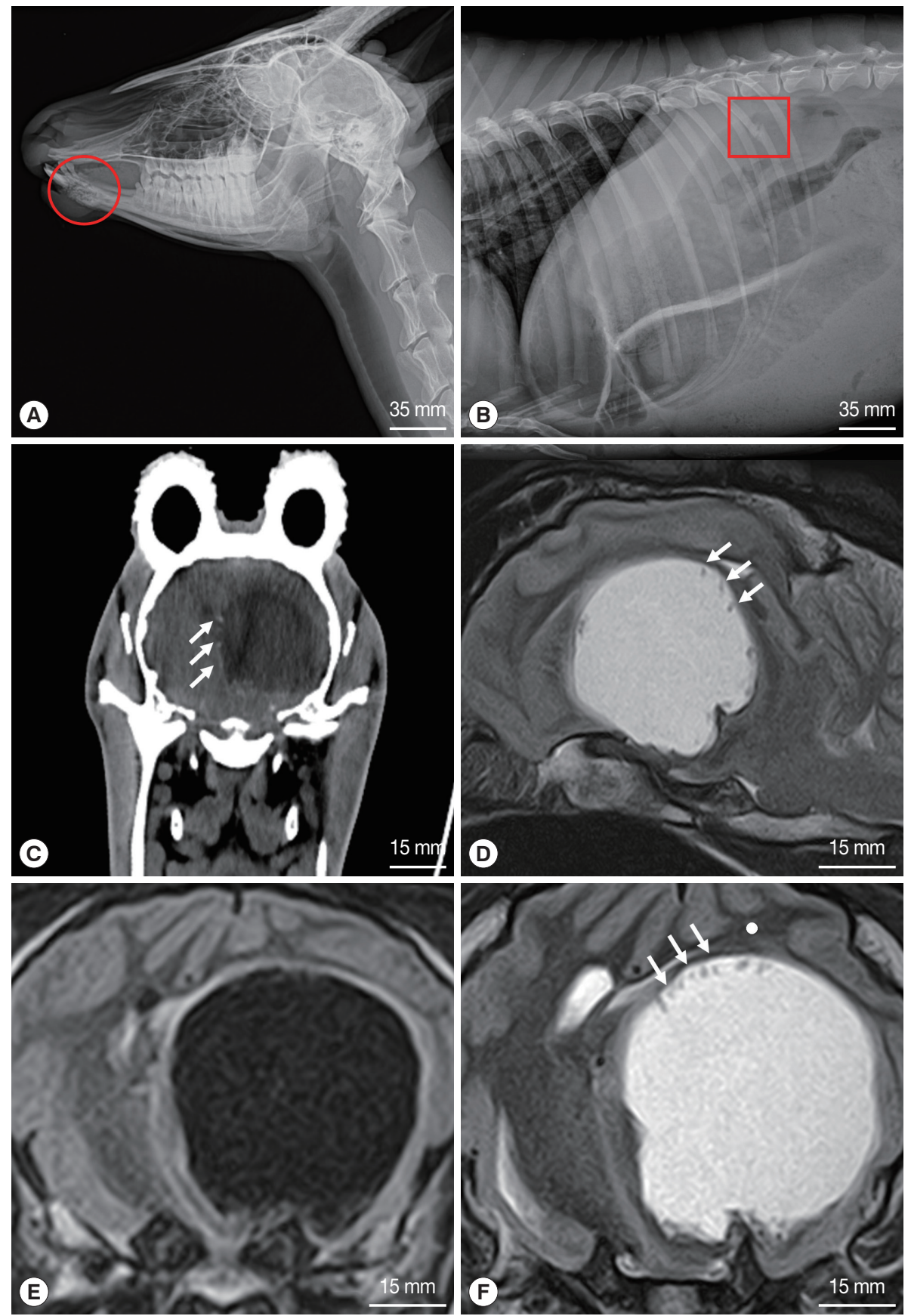

Fig. 1. Radiographs of the rescued goral. (A) osteolysis of the mandible (circle) and (B) fracture of the right 13th rib (square). CT and MRI of the brain. (C) Transverse CT shows a parasitic cyst located in the left cerebral hemisphere. (D) Sagittal MRI of the brain (T2-weighted). (E) Transverse T1 and (F) T2 weighted MRI. Note an enhanced rim on the periphery of the cyst in C, D, and F. white arrows indicate a protoscolex clusters.

Radiographs of the goral revealed osteolysis of the mandible, fracture of the right 13th rib, and severe pneumonia (Fig. $1 \mathrm{~A}, \mathrm{~B}$ ). Based on the radiographic findings, the goral was judged to have met a traffic accident (TA), and accordingly treatment was initiated, but the neurological symptoms per- sisted. CT and MRI of the brain were performed using standard procedures because the neurological symptoms did not improve despite treatment. The goral was positioned in sternal recumbency for CT and MRI examinations. CT scanning was performed using a Somatom Emotion 6 (Siemens, Erlangen, 

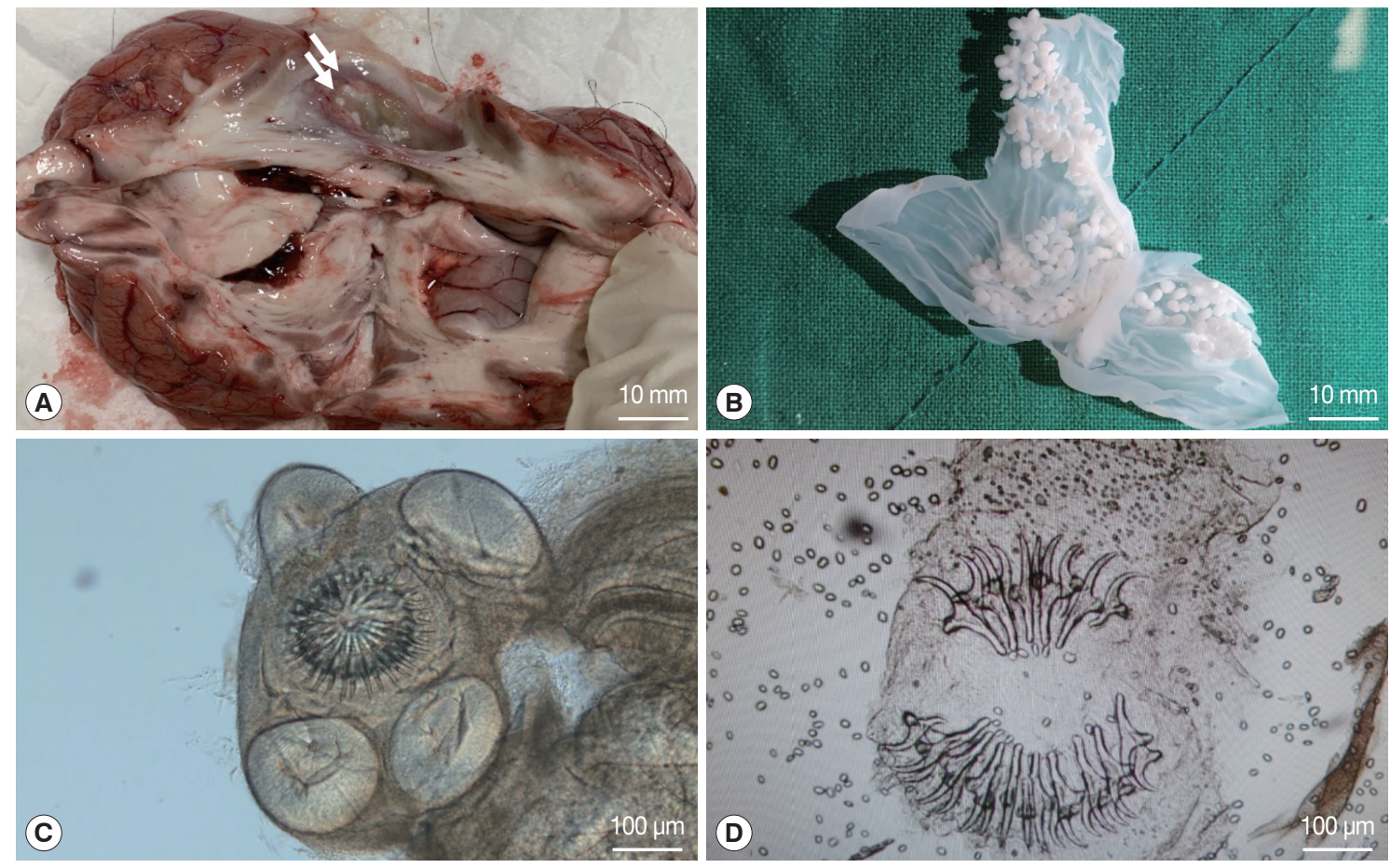

Fig. 2. Photographs of the Coenurus cerebralis cyst and protoscolex. (A) Coenurus cerebralis cyst in the left cerebral hemisphere. Multiple protoscolices can be seen as white spots on the inner surface of the cyst (arrows). (B) A ruptured cyst of Coenurus cerebralis removed from the brain of goral. (C) Protoscolex consisting of 4 suckers and a rostellum with hooks. (D) Two rows of large and small hooks with a total of 28 hooks.

Germany). MRI was performed by a $1.5 \mathrm{~T}$ unit (Vantage Elan ${ }^{\mathrm{TM}}$, Toshiba, Tokyo, Japan), using a combination of the 16 channel flexible coil (16-channel Flex SPEEDER Large ${ }^{\circledR}$, Toshiba, Tokyo, Japan) and spine coil (Octave SPEEDER Spine ${ }^{\circledR}$, Toshiba, Tokyo, Japan). In the CT images, the parasitic cyst $(3.1 \times 3.1$ $\mathrm{cm}$ ) was seen as a spherical structure with hypodensity in the left cerebral hemisphere (Fig. 1C). Faint hyperdense structures were also observed at the peripheral margin of the cyst, which were invaginated on the cyst wall (Fig. 1C). MRI of the head demonstrated the presence of a cystic structure in the brain parenchyma of the left cerebral hemisphere (Fig. 1D-F). The cyst was irregular, round shaped and clearly distinguished from the brain parenchyma. The cyst appeared hypointense in the T1 weighted (T1W) image (Fig. 1E) and hyperintense in the T2 weighted (T2W) image (Fig. 1D, F). In the T2W image, hypodense structures were found at the peripheral edge of the cyst inner wall (Fig. 1D, F), but not in the T1W image. Two days after MRI scan, the goral's clinical signs became severe with heavier breathing, shortness of breath, and eventually the goral died.

A post-mortem examination of the goral, which died due to worsening of symptoms, was performed. Examination of gross pathological lesions was performed. In that status, the cyst position was recorded and the size was measured using a caliper. When the skull was excised for the brain examination, a large amount of clear liquid flowed out. The cyst was transparent $3 \times 3 \mathrm{~cm}$ in size and was visible to the naked eye. In addition, the cyst detected in the left cerebral hemisphere was large, white and translucent with numerous protoscolices on the inner wall (Fig. 2A). When the cyst was evacuated from the brain, the affected part formed a large hollow from being under pressure for a long time. The cyst was ruptured in a petri dish and protoscolices were counted using a stereo microscope. The rice-shaped protoscolex attached to the internal layer of the cyst was isolated and observed under light microscope. The cyst contained a translucent fluid and approximately 320 protoscolices invaginating from the internal layer. Budding of numerous protoscolices from the internal layer of the cyst was seen as white clusters (Fig. 2B). The protoscolex had 4 suckers and a rostellum with hooks arranged in 2 rows (Fig. $2 \mathrm{C}$ ). The rostellum was arranged in 2 rows of large and small hooks with a total of 28 hooks (Fig. 2D). The average size of the large and small hooks was $157.5 \pm 2.6 \mu \mathrm{m}$ and $108.7 \pm 2.7$ $\mu \mathrm{m}$, respectively. 


\section{DISCUSSION}

Coenurus cerebralis is a metacestode of T. multiceps that infects the small intestine of carnivores $[10,11]$. The important intermediate hosts for T. multiceps are small ruminants such as sheep and goats [12], where oncospheres that infiltrate the brain or spinal cord develop into mature coenurus cysts in the central nervous system [13]. Most of the cysts of T. multiceps are detected on one side of the cerebral hemisphere, sometimes in the cerebellum [7]. In this study the cyst was found in the left cerebral hemisphere of a goral. A goral with neurological symptoms was rescued and died during treatment. As a result of postmortem examination, a parasitic cyst was found in the brain and identified as a larva of T. multiceps based on the following.

The characteristic clinical signs reported in animals infected with Coenurus cerebralis are dullness, depression, deviation of the head, circling, anorexia, head tilt and uncontrolled movement [7]. Until recently, interpretations of clinical signs and the sites of infection were one of the best ways to diagnose Coenurus cerebralis infection in small ruminants [14,15]. In this study, the clinical signs of the goral were similar to those reported in previous studies.

Other diagnostic methods such as CT and MRI have also been used to diagnose. CT was used in the diagnosis of coenurosis in humans and other animals. Several scholars have reported that the cyst appears to be a hypoattenuating structure with a mass effect in the CT images [10]. The MRI images of the brain parenchyma infected with Coenurus cerebralis appear hypointense in the T1W image and hyperintense in the T2W image [12]. CT and MRI are diagnostic methods that can accurately determine the exact location and size of the cyst. This information is essential for the surgical removal of the parasitic cysts [10]. In our case, hypointense cyst was detected in the left cerebral hemisphere on CT examination. In the T1W image, the cyst appeared hypointense, while in the T2W image it appeared hyperintense. In addition, the hypodense structures seen at the edge of the cyst inner wall on the CT and MRI images of gorals were determined to be protoscolices of C. cerebralis through post-mortem examination. To the best of our knowledge, in previous reported cases, protoscolices were frequently observed on CT, but not on MRI. However, protoscolices were observed in both MRI and CT in this study.

The Coenurus cerebralis cyst is readily recognized as a large, fluid filled cyst with numerous protoscolices attached to the inner wall [16]. The diameter of the cyst was wide, ranging between 0.8 and $9.0 \mathrm{~cm}[17,18]$. The number of protoscolices ranged between 40 and 550 per cyst [4]. Each protoscolex contained 26-28 hooks arranged in 2 rows of large and small hooks. The average total length of the large and small hooks was $157.7 \pm 0.5 \mu \mathrm{m}$ and $115 \pm 0.6 \mu \mathrm{m}$, respectively [15]. In our case, the diameter of the cyst $(3 \times 3 \mathrm{~cm})$, and the number of protoscolex (320), and the number and size of the hooks $(28 / 157.5 \times 108.7 \mu \mathrm{m})$ were all within the range recorded for Coenurus cerebralis infection caused by $T$. multiceps.

In conclusion, this is the first case report of goral coenurosis in Korea. Coenurosis is fatal in small ruminants and is an important public health parasitic disease as a zoonotic disease. In this study, the route of coenurosis infection in gorals has not been accurately identified, so continuous attention is required for several hosts.

\section{ACKNOWLEDGMENT}

This study was supported by the Kangwon National University in 2016.

\section{CONFLICT OF INTEREST}

The authors have no conflicts of interest concerning the work reported in this paper.

\section{REFERENCES}

1. Deplazes P, Eichenberger RM, Grimm F. Wildlife-transmitted Taenia and Versteria cysticercosis and coenurosis in humans and other primates. IJP: Parasites and Wildlife 2019; 9: 342-358. https:// doi.org/10.1016/j.ijppaw.2019.03.013

2. Oryan A, Moazeni M, Amrabadi O, Akbari M, Sharifiyazdi H. Comparison of distribution pattern, pathogenesis and molecular characteristics of larval stages of Taenia multiceps in sheep and goats. Small Ruminant Res. 2015; 132: 44-49. https://doi.org/10.1016/j.smallrumres.2015.10.008

3. Abera S, Wubit T, Nejash A. Cerebral coenurosis in small ruminants: a review. J Anim Sci Adv 2016; 6: 1595-1608. https://doi. org/10.5455/jasa.20160409121545

4. Rostami S, Beech RN, Salavati R, Baneshi MR, Kamyabi H, Harandi MF. Morphometric analysis of larval rostellar hooks in Taenia multiceps of sheep in Iran and its association with mitochondrial gene variability. Iran J Parasitol 2013; 8: 579-585.

5. Lescano AG, Zunt J. Other cestodes: sparganosis, coenurosis and Taenia crassiceps cysticercosis. Handb Clin Neurol 2013; 114: 335- 
345. https://doi.org/10.1016/B978-0-444-53490-3.00027-3

6. Collomb J, Machouart M, Biava MF, Brizion M, Montagne K, Plénat F, Fortier B. Contribution of NADH dehydrogenase subunit I and cytochrome C oxidase subunit I sequences toward identifying a case of human coenuriasis in France. J Parasitol 2007; 93: 934-937. https://doi.org/10.1645/GE-1160R.1

7. Sharma DK, Chauhan PPS. Coenurosis status in Afro-Asian region: a review. Small Ruminant Res. 2006; 64: 197-202. https:// doi.org/10.1016/j.smallrumres.2005.05.021

8. Sivakumar S, Schuster RK, Wieckowsky T. Non-cerebral coenurosis in goats. Parasitol Res 2010; 107: 721-726. https://doi.org/10.1007/ s00436-010-1919-6

9. Myslenkov AI, Voloshina V. Ecology and Behaviour of the Amur Goral. Nauka, Moscow. 1989, pp 1-128.

10. Gazioglu A, Simsek S, Kizil O, Ceribasi AO, Kesik HK, Ahmed H. Clinical, pathological and molecular evaluations and CT scan screening of coenurosis (Coenurus cerebralis) in sheep and calves. Braz J Vet Parasitol Jaboticabal 2017; 26: 3-9. https://doi.org/ 10.1590/s1984-29612016090

11. Gómez M, Tadich N, Mieres M, Bustamante H, Galecio J, Hervé $\mathrm{M}$. Computed tomographic findings in chronic cerebral coenurosis associated with secondary hydrocephalus in a young ewe. Arch Med Vet 2007; 39: 281-285.

12. Sarma BK, Das J. Surgical management of cerebral coenurosis of goats in Lakhimpur district of Assam (India). J Entomol Zool
Stud 2019; 7: 1268-1270.

13. Varcasia A, Pipia AP, Dessì G, Zidda A, Tamponi C, Pau M, Scala A, Boufana B. Morphology and genetic variability within Taenia multiceps in ruminants from Italy. Vet Parasitol 2016; 223: 181185. https://doi.org/10.1016/j.vetpar.2016.04.039

14. Gonzalo-Orden JM, Altónaga JR, Diez A, Gonzalo JM, Orden MA. Correlation between MRI, computed tomographic findings and clinical signs in a case of ovine coenurosis. Vet Rec 2000; 146: 352-353. https://doi.org/10.1136/vr.146.12.352

15. El-Neweshy MS, Khalafalla RE, Ahmed MMS, Al Mawly JH, ElManakhly EM. First report of an outbreak of cerebral coenurosis in Dhofari goats in Oman. Braz J Vet Parasitol Jaboticabal 2019; 28: 479-488. https://doi.org/10.1590/s1984-29612019054

16. Merbl Y, Shilo-Benjamini Y, Chai O, Chamisha Y, Anglister N, King R, Horowitz I, Aizenberg Z, Shamir MH. Taenia multiceps brain cyst removal in two wild Nubian ibex (Capra nubianas). J Zoo Wildl Med 2014; 45: 193-196. https://doi.org/10.1638/20130175R.1

17. Yoshino T, Momotani E. A case of bovine coenurosis (Coenurus cerebralis) in Japan. Jpn J Vet Sci 1988; 50: 433-438. https://doi. org/10.1292/jvms1939.50.433

18. Achenef M, Markos T, Feseha G, Hibret A, Tembely S. Coenurus cerebralis infection in Ethiopian highland sheep: Incidence and observations on pathogenesis and clinical signs. Trop Anim Health Pro 1999; 31: 15-24. https://doi.org/10.1023/a:1005125316275 
